\title{
EFFICIENCY OF HYSTERETIC DAMPER IN OSCILLATING SYSTEMS
}

\author{
M.E. Semenov ${ }^{1,2,3,4, *}$, Andrey M. Solovyov ${ }^{1}$, \\ Peter A. Meleshenko ${ }^{1,5}$ and Olga O. Reshetova ${ }^{1}$
}

\begin{abstract}
This paper is dedicated to comparative analysis of nonlinear damping in the oscillating systems. More specifically, we present the particular results for linear and nonlinear viscous dampers, fractional damper, as well as for the hysteretic damper in linear and nonlinear (Duffing-like) oscillating systems. We consider a constructive mathematical model of the damper with hysteretic properties on the basis of the Ishlinskii-Prandtl model. Numerical results for the observable characteristics, such as the force transmission function and the "force-displacement" transmission function are obtained and analyzed for both cases of the periodic affection, as well as for the impulse affection (in the form of $\delta$-function). A comparison of an efficiency (in terms of the corresponding transmission functions) of the nonlinear viscous damper and the hysteretic damper is also presented and discussed.
\end{abstract}

Mathematics Subject Classification. 34G20, 34C55, 47J40

Received December 15, 2018. Accepted November 19, 2019.

\section{INTRODUCTION}

The dampers and damping processes have a long history and especially relevant (from both the fundamental and the applied points of view) in the present days due to development of the modern impact-vibrational systems (see, e.g., [1]). The damper is a device used for damping the mechanical, electrical and other modes of vibration arising in the machines and mechanical systems during its operation. Damping is an important task that has a wide range of applications. In general the damping is a process whereby the energy is taken from the vibrating system and is being absorbed by the surroundings. The examples of damping include internal forces of a spring, viscous force in a fluid, electromagnetic damping in galvanometers, shock absorber in a car, anti seismic damping devices in buildings, etc. At the same time it should be noted that the damping devices are widely used in modern avionics (damper of aero-elastic vibrations, which is the electronic system for automatic cancellation of short-aircraft vibrations which inevitably arise when the flight modes change). Let us also list

Keywords and phrases: Linear and nonlinear damping, fractional damper, hysteretic damper, hysteron, Ishlinskii-Prandtl model.

1 Voronezh State University, Universitetskaya sq. 1, 394018, Voronezh, Russia

${ }^{2}$ Geophysical Survey of Russia Academy of Science, Lenina av. 189, 249035 Obninsk, Russia

3 Voronezh State Technical University, XX-letiya Oktyabrya st. 84, 394006 Voronezh, Russia

4 Zhukovsky-Gagarin Air Force Academy, Starykh Bolshevikov st. 54 "A", 394064 Voronezh, Russia

5 Target Search Lab of Groundbreaking Radio Communication Technologies of Advanced Research Foundation, Plekhanovskaya st. 14, 394018, Voronezh, Russia

* Corresponding author: mkl150@mail.ru 
the types (relative to the physical nature of damping process) of damping devices, namely: structural, Coulomb friction, elastomer, active drivers, passive hydraulic, semi-active hydraulic, adaptive hydraulic, etc.

In the case of mechanical oscillations the model of linear viscous damping (which is based on the energy dissipation due to viscous friction) is widely used. However, this type of damping has a significant drawback, namely the low efficiency outside the region of resonance of the system. One way to solve this problem is to use a nonlinear viscous damper $[4,5,8,11,12,16-18,29]$ or a damper with hysteretic properties [9, 28] (here we should note the recent work [13] where the effects of nonlinear hysteretic damping on the post-critical behavior of the viscoelastic Beck's beam are discussed). Particularly, in the well-known book [17] different forms of nonlinear damping (together with several examples) are presented and discussed completely especially from the point of view their effects on the nonlinear behavior of dynamical systems. In $[5,12,14]$ the destabilizing effect of nonlinear damping on the post-critical behavior of non-conservative discrete systems (Ziegler's systems) is discussed and analyzed. Let us note also interesting works dedicated to experiments with the hysteretic damping systems (see, e.g., [28] and related references, as well as the book [9]). The influence of nonlinear damping effects on the stability of oscillating systems are considered in [14] (see, also the related references). It should also be pointed out the exciting and interesting (generally, from the fundamental point of view) model of generalized viscous damping which is based on the technique of fractional derivatives $[2,10,30]$. An interest to the generalized viscous damping is connected with the fact that the systems with such a type of nonlinearity demonstrate a chaotic behavior [26]. Also the generalized viscous damping can be considered as an effective model for the so-called visco-elastic materials which describe the properties of all the materials in the extremely natural way (because there are no pure elastic materials, as well as there are no pure viscous materials; all the materials have both the viscous and elastic properties that can be observed at various motion conditions).

Main purpose of this work is to study the dynamics of a mechanical system (oscillating mechanical system) under various external affections (forced oscillations) in the presence of a damping block. Special interest in this case has a damper with hysteretic properties. As a mathematical model of such a hysteretic damper we consider the Ishlinskii-Prandtl "converter" [19,21], which is a type of continual hysterons, and can be presented as a system of parallel coupled nonlinear links such as "stops" (hysterons) [7]. Here we would like to note that the mathematical models of various hysteretic system was considered in a number of works (see, e.g., [6, 15, 20, 22, 25] and related references). However, in these works authors use the phenomenological models of hysteresis (such as the Bouc-Wen model, the Duhem-type modesl, etc.). In this paper we use the design model of hysteresis, namely, the operator technique of Krasnosel'skii-Pokrovskii (see [23, 24]) which has an exact physical meaning in comparison with the phenomenological models. Thus, in this paper we propose and analyze the efficiency (in terms of the corresponding transmission functions) of the hysteretic damping model based on the IshlinskiiPrandtl material. Particularly, in contrast to early works on the hysteretic damping, in the proposed model we use the design approach to hysteresis converter.

\section{DAMPING}

In this section we describe the physical model of mechanical system with the damping device. Also, in this section we present some results (namely the equations of motion, as well as some observable peculiarities) for various kinds of damping, namely the linear and nonlinear viscous damping [17], fractional damping [3, 27, 31], and hysteretic damping.

\subsection{Physical system}

Let us consider a mechanical system under external affection in the presence of damping part $f_{d}(t)$ as is shown in Figure 1. The system is presented as a cylinder with mass $M$ under external affection $f(t)$ of harmonic nature. In the cylinder there is a car of mass $m$ (moving without friction in the horizontal plane) connected to the border by a spring with stiffness $k$ (linear, or nonlinear as well). For simplicity we assume that the system is one-dimensional. 


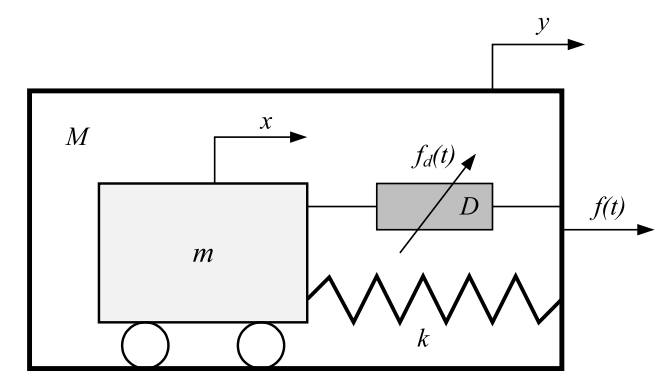

FiguRE 1. The considered physical system.

Let us suppose that the external affection $f(t)$ is described by the following relation (harmonically changes with time):

$$
f(t)=Y \omega^{2} \sin (\omega t),
$$

where $Y \omega^{2}$ is an amplitude and $\omega$ is a frequency of the affection.

The equation of motion for considered system is

$$
\left\{\begin{array}{c}
M \ddot{y}+k z+f_{d}(t)=f(t) \\
m \ddot{x}-k z-f_{d}(t)=0 \\
f(t)=Y \omega^{2} \sin (\omega t), z(t)=y(t)-x(t) .
\end{array}\right.
$$

Here overdot means the time derivative and $z(t)$ is a relative displacement.

\subsection{Linear and nonlinear viscous damping}

Let us consider the case of viscous damping. In general case the viscous friction can be described as follows:

$$
f_{d}(t)=c(1+z(t))^{n} \dot{z}, n \geqslant 0,
$$

where $c$ is a damping coefficient. In the case $n=0$ we have a linear viscous friction. For $n>0$ the nonlinear viscous friction takes place $[11,16]$.

The equation of motion for relative displacement $z(t)$ becomes

$$
\ddot{z}(t)+\frac{M+m}{M m}\left\{c[1+z(t)]^{n} \dot{z}(t)+k z(t)\right\}=\frac{Y}{M} \omega^{2} \sin (\omega t) .
$$

It is more suitable to introduce the dimensionless variables

$$
\begin{gathered}
\Omega=\frac{\omega}{\omega_{0}}, \mu=\frac{m M}{m+M}, A=\frac{Y}{M}, \tau=\omega_{0} t \\
\omega_{0}=\sqrt{\frac{k}{\mu}}, \zeta=\frac{c}{2 \omega_{0} \mu}, u=z .
\end{gathered}
$$

After such notations the final equation of motion has the form:

$$
\frac{\mathrm{d}^{2} u}{\mathrm{~d} \tau^{2}}+2 \zeta(1+u)^{n} \frac{\mathrm{d} u}{\mathrm{~d} \tau}+u=A \Omega^{2} \sin (\Omega \tau)
$$




\subsection{Fractional damping}

The so-called fractional damping $[3,27]$ is described by the following relation:

$$
f_{d}(t)=\delta \dot{z}(t)|\dot{z}(t)|^{p-1}
$$

where $\delta$ is a damping coefficient, and $p$ is a positive parameter. As it can be seen from this relation the fractional damping term turns into Coulomb friction (Coulomb damping) at $p \rightarrow 0$, while at $p=1$ it turns to a linear viscous friction (linear viscous damper).

The equation of motion in the case of fractional damping turns into:

$$
\ddot{z}(t)+\frac{M+m}{M m}\left\{\delta \dot{z}(t)|\dot{z}(t)|^{p-1}+k z(t)\right\}=\frac{Y}{M} \omega^{2} \sin (\omega t) .
$$

In addition, let us consider a case where restoring force of the spring can be presented in the form:

$$
f_{k}(t)=k_{0} z(t)+k_{1} z^{3}(t)
$$

This restoring force corresponds to nonlinear spring (out of Hooke's law). In this case the equation of motion has the following form:

$$
\ddot{z}(t)+\frac{M+m}{M m}\left\{\delta \dot{z}(t)|\dot{z}(t)|^{p-1}+k_{0} z(t)+k_{1} z^{3}(t)\right\}=\frac{Y}{M} \omega^{2} \sin (\omega t) .
$$

Introducing the dimensionless parameters

$$
\omega_{0}^{2}=\frac{k_{0}}{\mu}, \beta \omega_{0}^{2}=\frac{k_{1}}{\mu}, \frac{\alpha}{\omega_{0}^{p-2}}=\frac{\delta}{\mu}
$$

the equation of motion turns into a Duffing-like equation with the fractional damping term:

$$
\frac{\mathrm{d}^{2} z}{\mathrm{~d} \tau^{2}}+\alpha \frac{\mathrm{d} z}{\mathrm{~d} \tau}\left|\frac{\mathrm{d} z}{\mathrm{~d} \tau}\right|^{p-1}+z(\tau)+\beta z^{3}(\tau)=A \Omega^{2} \sin (\Omega \tau)
$$

Below we compare this type of damping and the hysteretic damping using the corresponding transmission functions.

\subsection{Hysteretic damping}

\subsubsection{Hysteretic Ishlinskii-Prandtl material}

The materials which are used for hysteretic dampers in general are polymers (synthetic rubber). The composition of such materials is chosen appropriately to provide the high damping properties in a specific range of frequencies and temperatures. When the material is damped the energy dissipation occurs within the material itself. Such an effect is caused by friction between the inner layers that are "flow" when the damping occurs. In the case when such a structure is under damping vibrations the hysteresis loop appears in the "stress-strain". The area of the loop determines the energy loss (per unit volume of the body) in one cycle due to damping.

Let us briefly consider a mathematical model of hysteresis (based on the operator technique). The carrier of hysteretic nonlinearities is a converter $W$ with scalar input $u(t)$ and outputs $x(t)$. The state of this converter is a pair $\{u, x\}$, i.e., an input-output pair. Let the set of possible states of the converter $W$ is a strip $\Omega=\Omega(W)$ which is placed between the two horizontal lines $\Phi_{l}$ and $\Phi_{r}$, as is shown in Figure 2. 


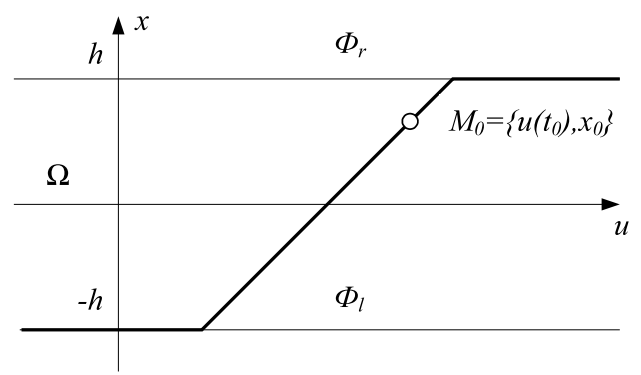

Figure 2. Action of hysteron-nonlinearity.

If the input $u(t)\left(t \geqslant t_{0}\right)$ is continuous and monotone then the output can be defined as:

$$
x(t)=W\left[t_{0}, x_{0}\right] u(t)(t \geqslant t 0)
$$

so that the variable state $\{u(t), x(t)\}$ is a point in the broken line as is shown in Figure 2 by thick line; this broken line (passing through the initial state $M_{0}=\left\{u\left(t_{0}\right), x_{0}\right\}$ and the ends on the lines $\Phi_{l}$ and $\Phi_{r}$ ) consists of segment with slope 1 and two horizontal half-lines. In other words, when the input is monotonic the output is determined by the equation:

$$
x(t)=\left\{\begin{array}{l}
\min \left\{h, u(t)-u\left(t_{0}\right)+x\left(t_{0}\right)\right\}, u(t) \text { nondecreasing } \\
\max \left\{-h, u(t)-u\left(t_{0}\right)+x\left(t_{0}\right)\right\}, u(t) \text { nonincreasing. }
\end{array}\right.
$$

To determine the output (2.9) for piecewise monotonic continuous inputs we can use the semigroup identity

$$
W\left[t_{0}, x_{0}\right] u(t)=W\left[t_{1}, W\left[t_{0}, x_{0}\right] u\left(t_{1}\right)\right] u(t)\left(t_{0} \leqslant t_{1} \leqslant t\right) .
$$

The described converter is called as a "stop" or a hysteron. With the special limit construction such a converter can be defined on all the monotonic inputs [7].

In the most common models of elastic-plastic fibers their states are completely determined by the variables of deformation $u$ and stress $x$. The parameter $h$ in this case is called as the yield strength of the material. Such fibers can be considered as converters with an input in the form of deformation and an output in the form of strain. In the Prandtl model the strain is determined by the deformation in the same manner as in hysteron-nonlinearity, but the trajectories of possible states between the boundary horizontal lines have the slope $E$ which differs from 1 (for small deformations the fiber can be considered as an elastic material with the elastic modulus $E$ ).

Let us consider the inverter $W$ which can be presented in the form of a simple flow diagram (without feedback) based on the finite number of hysterons $W^{1}, \ldots, W^{N}$ and elementary functional units (as is shown in Fig. 3). Usually such converters $W$ are non-deterministic. Their condition can be described (instead of the input-output description) by the set $\left\{u, z_{1}, \ldots, z_{N}\right\} \in R^{N-1}$, where $u$ is the converter's input and $z_{j}$ is an output of hysteron $W^{j}$ in the flow diagram.

Let us suppose that the hysterons $W^{1}, \ldots, W^{N}$ with areas of permissible states $\Omega\left(W^{1}\right), \ldots, \Omega\left(W^{N}\right)$ are determined by the input-output relations

$$
z_{j}(t)=W^{j}\left[t_{0}, z_{j}\left(t_{0}\right)\right] u(t)(j=1, \ldots, N)
$$

Let

$$
\Omega(W)=\left\{\left\{u, z_{1}, \ldots, z_{N}\right\}:\left\{u, z_{j}\right\} \in \Omega\left(W^{j}\right), u \in R^{1}\right\} .
$$




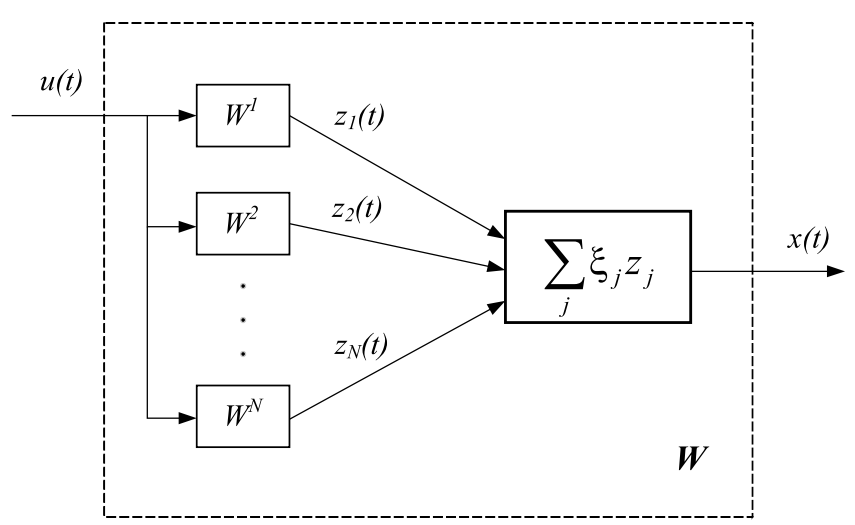

Figure 3. Parallel connection of hysterons.

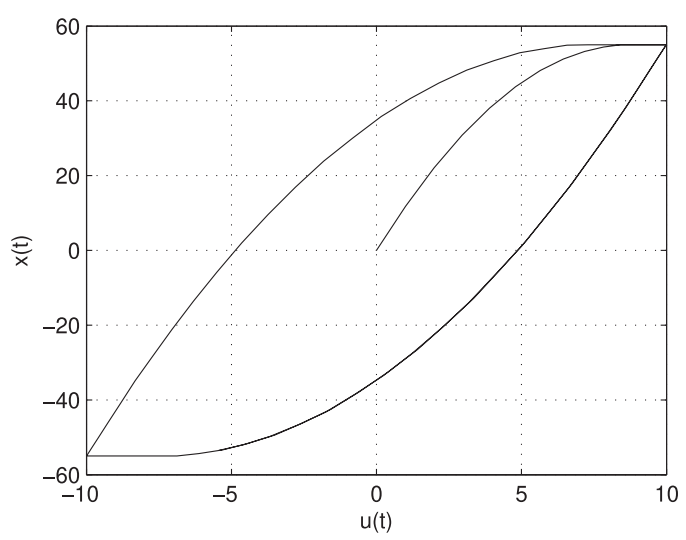

FIGURE 4. "Stress-strain" diagram for Ishlinskii-Prandtl material.

Parallel connection of hysterons $W^{j}$ with weights $\xi_{j}$ we call the converter $W$ with an area of permissible states (2.13) such that for every initial state

$$
q\left(t_{0}\right)=\left\{u_{0}, z_{0}\right\}=\left\{u\left(t_{0}\right), z_{1}\left(t_{0}\right), \ldots, z_{N}\left(t_{0}\right)\right\} \in \Omega(W) \subset R^{N+1}
$$

all the continuous scalar inputs $u(t)\left(t \geqslant t_{0}\right)$ that satisfy the condition $u\left(t_{0}\right)=u_{0}$ are allowed. The output is determined by the relation

$$
x(t)=W\left[t_{0}, z_{0}\right] u(t)=\sum_{j=1}^{N} \xi_{j} W^{j}\left[t_{0}, z_{j}\left(t_{0}\right)\right] u(t),\left(t \geqslant t_{0}\right) .
$$

The considered converter $W$ is called as an Ishlinskii-Prandtl material [32]. Of course the "classical" IshlinskiiPrandtl converter is determined as a continuos system (sum should be replaced by an integral). However in our numerical simulations it is more appropriate to use the discrete analog of the Ishlinskii-Prandtl converter (2.15). In this way in the following consideration we call the converter (2.15) as an Ishlinskii-Prandtl converter.

As an example we consider the reaction of the Ishlinskii-Prandtl material on a sinusoidal impact. We use the converter $W$ with the following parameters: $E=1, W^{j}\left[t_{0}, z_{j}\left(t_{0}\right)\right]=0, \xi_{j}=1, W^{j}: h=\{-j, j\}, j=1, \ldots, 10$ and the input in the form $u(t)=12 \sin (t)$. "Stress-strain" diagram of such a converter is shown in Figure 4. 


\subsubsection{Hysteretic damping}

Now we consider the hysteretic damper. In this case (using the notations introduced above) the damping force can be presented as:

$$
f_{d}(\tau)=W\left[\tau, z_{j}(\tau)\right] u, j=\overline{1, N},
$$

where $W$ is an Ishlinskii-Prandtl operator which is determined by the relation (2.15).

In this case the equation of motion for the considered system becomes:

$$
\frac{\mathrm{d}^{2} u}{\mathrm{~d} \tau^{2}}+\eta W\left[\tau, z_{j}(\tau)\right] u+u=A \Omega^{2} \sin (\Omega \tau), j=\overline{1, N},
$$

where $\eta=S / k$ and $S$ is an area of damping material's section, $k$ is a spring stiffness.

In the case of a nonlinear spring (taking into account the cubic term in the expansion of restoring force) the corresponding equation of motion becomes:

$$
\frac{\mathrm{d}^{2} z}{\mathrm{~d} \tau^{2}}+\gamma W\left[\tau, z_{j}(\tau)\right] z(\tau)+z(\tau)+\beta z^{3}(\tau)=A \Omega^{2} \sin (\Omega \tau), j=\overline{1, N}
$$

where $\gamma \omega_{0}^{2}=\frac{\eta}{\mu}$, and the corresponding parameters of the converter are described above.

\section{Numerical Simulation}

\subsection{Transmission characteristics}

Let us consider the main characteristics reflecting the efficiency of the damper in the resonance region and beyond. Such characteristics are the force transmission function and the "force-displacement" transmission function.

The force transmission function is determined by the ratio of the force applied to the cylinder $M$ and the force applied to the car $m$ (Fig. 1). This function reflects the efficiency of suppression of the external affection by the force transmission from an external source to the load. This characteristic is expressed as follows:

$$
T_{f f}=\frac{1}{Y \omega^{2}} \max \left|m \omega_{0}^{2} \frac{\mathrm{d}^{2} x}{\mathrm{~d} \tau^{2}}\right|
$$

In the case of viscous damper, using the equations (2.4) and (2.5) we can express the characteristic $T_{f f}$ through the variable $u$, namely:

$$
T_{f f}=\max \left|\frac{m}{(m+M) A \Omega^{2}}\left[A \Omega^{2} \sin (\Omega \tau)-\ddot{u}\right]\right| .
$$

The "force-displacement" transmission function is determined by the relation of the motion of car $m$ relative to the cylinder $M$ and the force applied to the cylinder. This quantity reflects the efficiency of vibration absorption by the ability of the damper to reduce the relative motion of the car under influence of external forces. This characteristic is expressed as

$$
T_{f d}=\frac{\max |x(\tau)|}{Y \omega^{2}}
$$




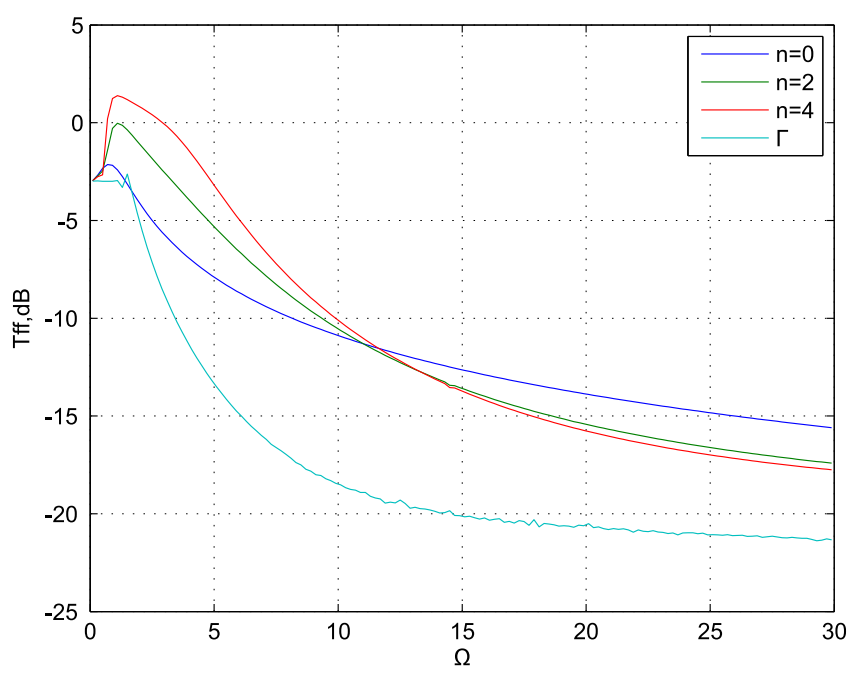

FiguRE 5. Force transmission function.

As previously, in the case of viscous damping, using the equations (2.4) and (2.5) we can express the $T_{f d}$ function in the following form:

$$
T_{f d}=\max \left|\frac{A \sin (\Omega \tau)+u}{(m+M) A \Omega^{2} \omega_{0}^{2}}\right| .
$$

During the following simulations we use these quantities for comparison of the linear viscous, nonlinear viscous, fractional and hysteretic dampers.

\subsection{Simulation results}

We make the numerical simulations using the explicit difference schema. In order to compare the viscous damper and hysteretic damper we present the numerical results for two characteristics of the system, namely, the force transmission function and the "force-displacement" transmission function. For the nonlinear viscous damper we use the following set $n=\{0,2,4\}$.

For the hysteretic damper we use the Ishlinskii-Prandtl material (which corresponds to rubber) with parameters $E=10000, W^{j}\left[t_{0}, z_{j}\left(t_{0}\right)\right]=0, \xi_{j}=1, W^{j}: h=\{-j, j\}, j=1, \ldots, 50$ (with a step 0.1 ), $\gamma=0,0001$.

The characteristics of the mechanical system (per dimensionless units): $M=1, m=1, \zeta=0.8, \omega_{0}=10$; the external affection with parameters $A=1, \omega=0, \ldots, 30$ (with a step 0.2); parameters of the difference scheme: $h_{\tau}=0.0167$ (a step of a grid by the time axis), $T=10000$ (a time period corresponding to modelling time).

The simulation results are shown in Figures 5 and 6 (these results correspond to a linear restoring force of the spring). As it can be seen from these figures the linear viscous damper (dark blue curve $n=0$ ) has a high efficiency in the resonance region of the system, however outside the resonance region the damping properties sharply decrease. At the same time the nonlinear viscous damper (green $n=2$ and red $n=4$ curves) has a wide range of effective use, but loses in efficiency to linear damper in the resonance region of the system.

The hysteretic damper (light blue curve) based on Ishlinskii-Prandtl material has a high efficiency both in the resonance region and beyond. The disadvantage of the hysteretic damper is in decreasing of the ability to reduce a relative movement of a car under external forces outside the resonance region of the system.

Let us consider also the phase portraits for the system both for the cases of viscous damper and for hysteretic damper. As the origin of the phase plane we use the instantaneous values of the car's coordinate inside the cylinder $x(\tau)$ and its relative speed $\dot{x}(\tau)$. For the case of viscous damper (for the hysteretic damper we can use 


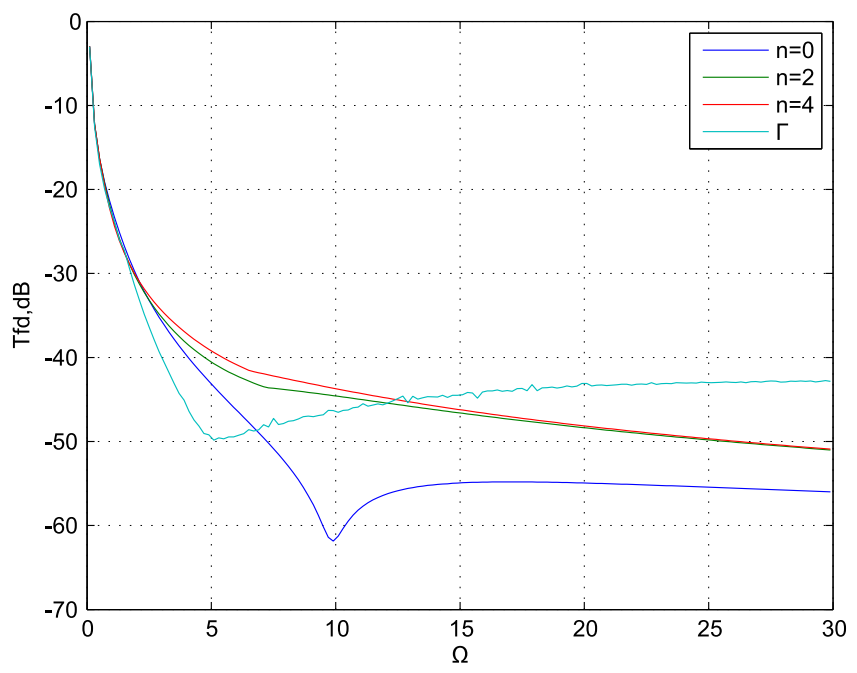

FIGURE 6. "Force-displacement" transmission function.
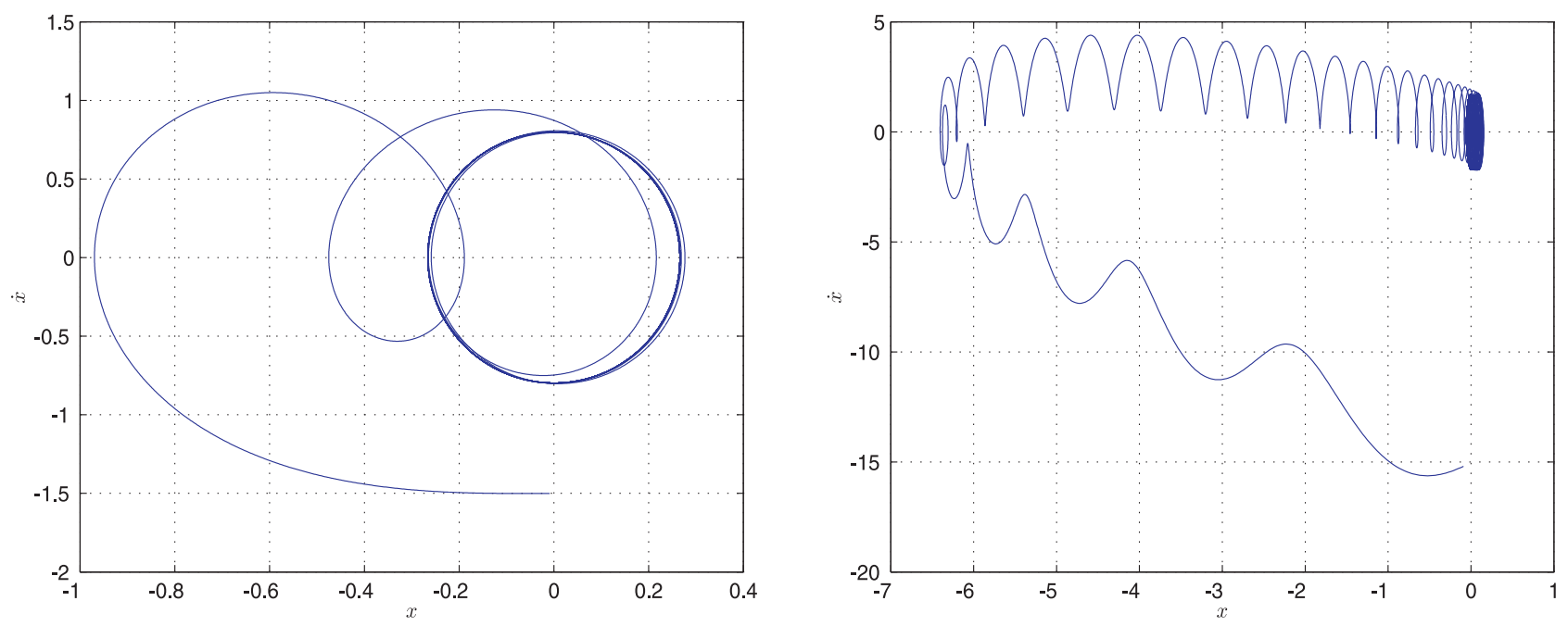

Figure 7 . Phase portraits of the system in the case of linear viscous damper $(n=0)$ at $\Omega=3$ (left panel) and $\Omega=30$ (right panel).

the numerical solutions only), using the equations (2.4) and (2.5) we can write (taking into account the general system of equations (2.2) together with the corresponding notations):

$$
\begin{gathered}
x(\tau)=-[A \sin (\Omega \tau)+u] \frac{M}{M+m}, \\
\dot{x}(\tau)=-[A \Omega \cos (\Omega \tau)+\dot{u}] \frac{M}{M+m} .
\end{gathered}
$$

The phase portraits of the system under consideration (with various kinds of dampers) are presented in the figures $7-10$ 

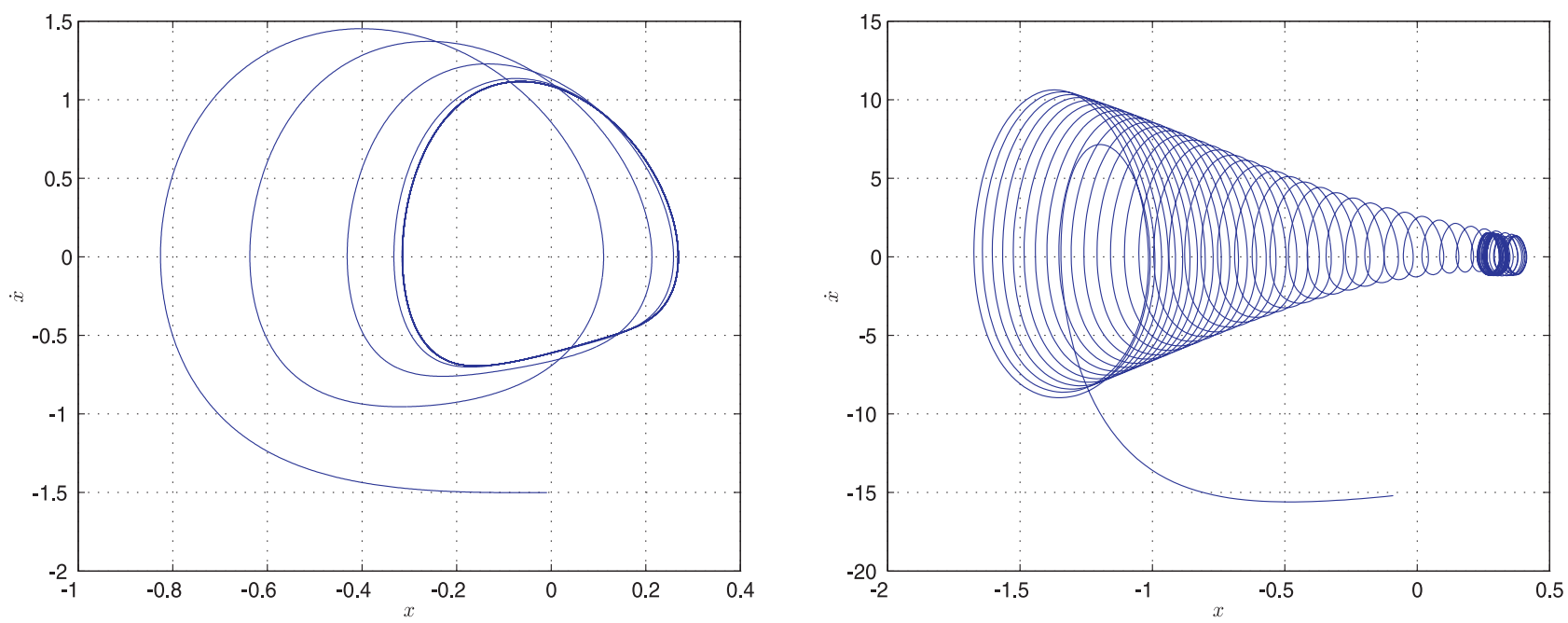

FiguRE 8. Phase portraits of the system in the case of nonlinear viscous damper $(n=2)$ at $\Omega=3$ (left panel) and $\Omega=30$ (right panel).
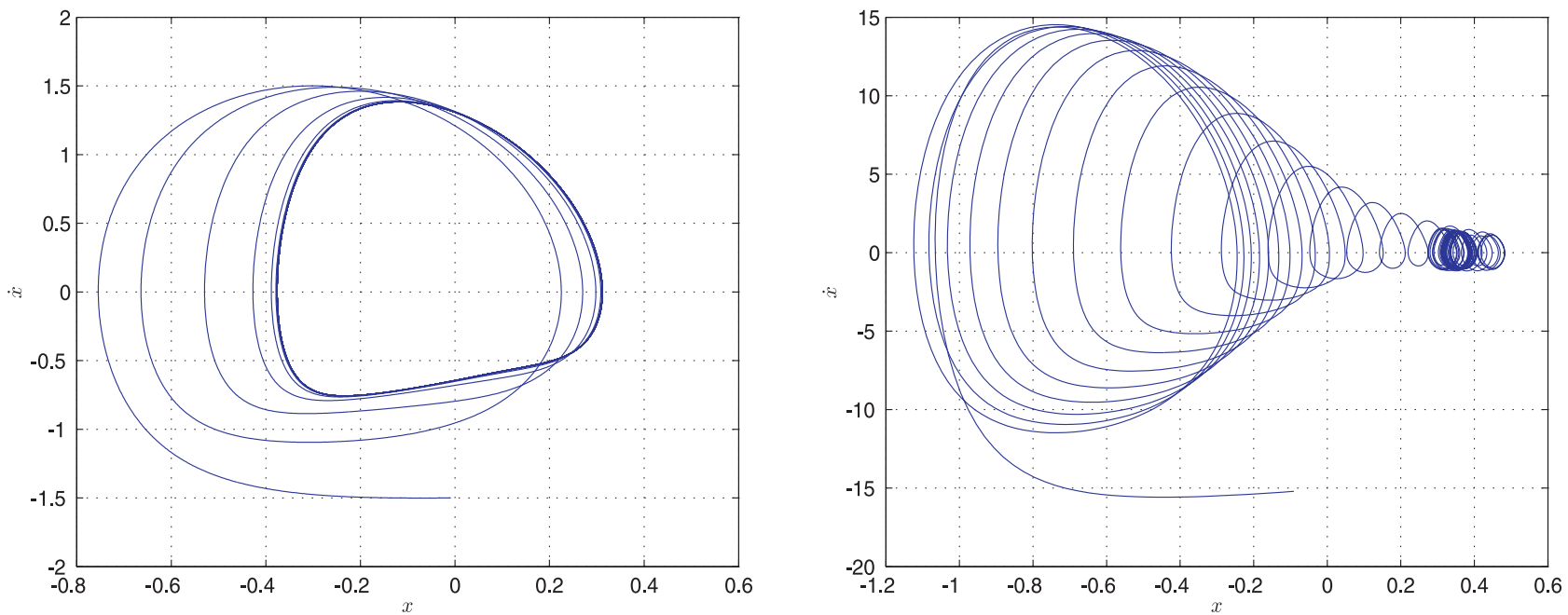

Figure 9. Phase portraits of the system in the case of nonlinear viscous damper $(n=4)$ at $\Omega=3$ (left panel) and $\Omega=30$ (right panel).

Figures show the phase portraits of the system both in the case of viscous (linear and nonlinear) damping and the hysteretic damping (a linear restoring force of the spring). The left panels show the phase portraits in the resonance region, the right panels show the far frequency domain. From the figures it is clear that the hysteretic damper has a greater efficiency (in comparison with the linear and nonlinear viscous dampers) in the resonance region, and beyond. Previously, based on the analysis of transmission functions $T_{f f}$ and $T_{f d}$ we have made some conclusions on the efficiency of hysteretic damper in comparison with the viscous damper. In this way the presented phase portraits are proved our conclusions.

Let us compare also an efficiency of hysteretic and fractional dampers (in the same way, using the transmission functions) in the case of a nonlinear restoring force of the spring. In Figure 11 and 12 we demonstrate the 

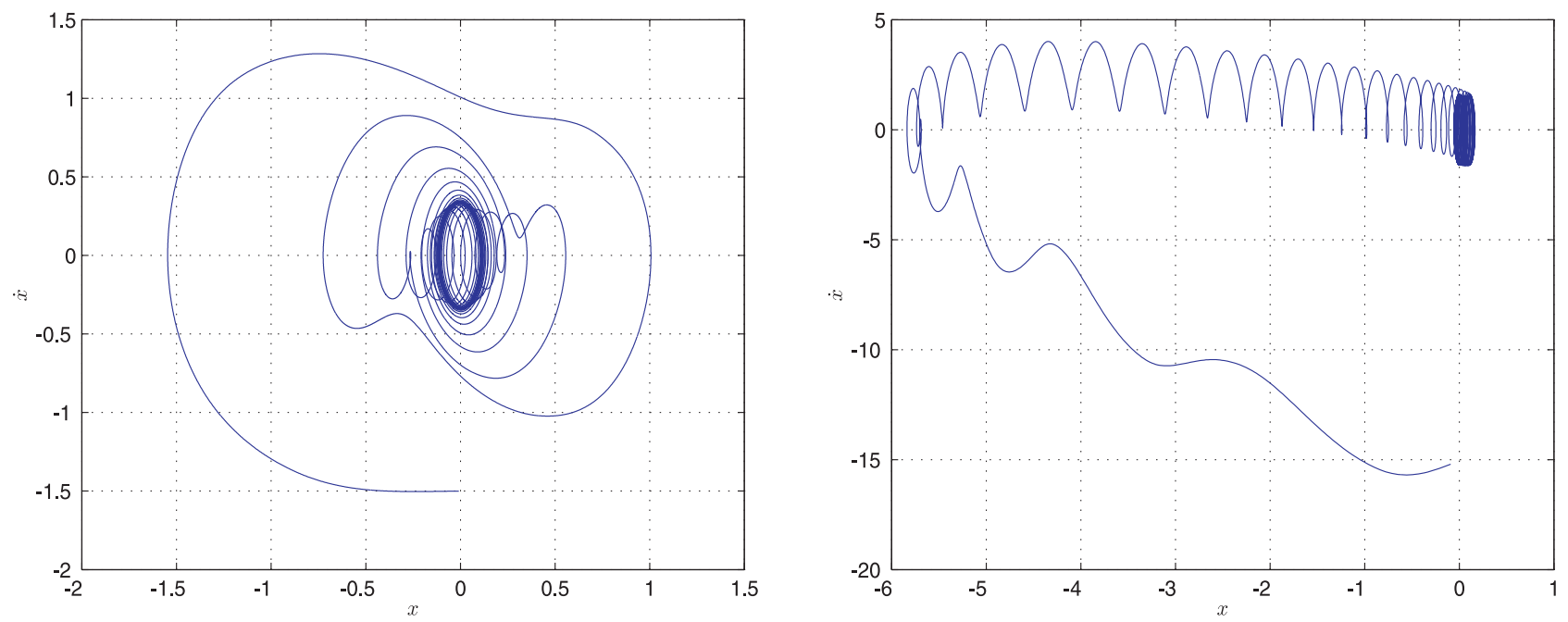

FiguRE 10 . Phase portraits of the system in the case of hysteretic damper at $\Omega=3$ (left panel) and $\Omega=30$ (right panel).

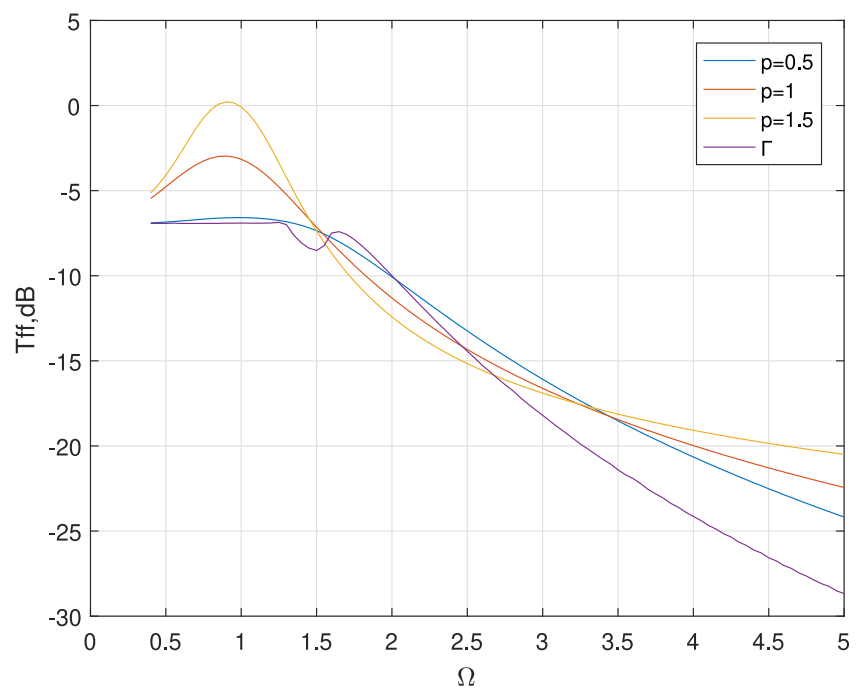

FIGURE 11. Force transmission function in the case of Duffing-like equation with fractional damping (2.8) and hysteretic damping (2.18) (a coefficient at the cubic term is $\beta=1$ ).

numerical results for observable characteristics of the corresponding mechanical system described by the Duffinglike equations (2.8) and (2.18) (parameters $\alpha$ and $\gamma$ are: $\alpha=1, \gamma=1$; an "intensity" of the cubic term is $\beta=1$ ).

As it can be seen from these figures the hysteretic damper also demonstrate a high efficiency (in terms of observable characteristics such as transmission functions) in comparison with the fractional damper for various quantities of parameter $p$.

In the final step, let us study the dynamics of the system under a pulse affection (we consider a single pulse in the form of $\delta$-function). In this case, the equation (2.4) for the viscous damper can be rewritten in the following 


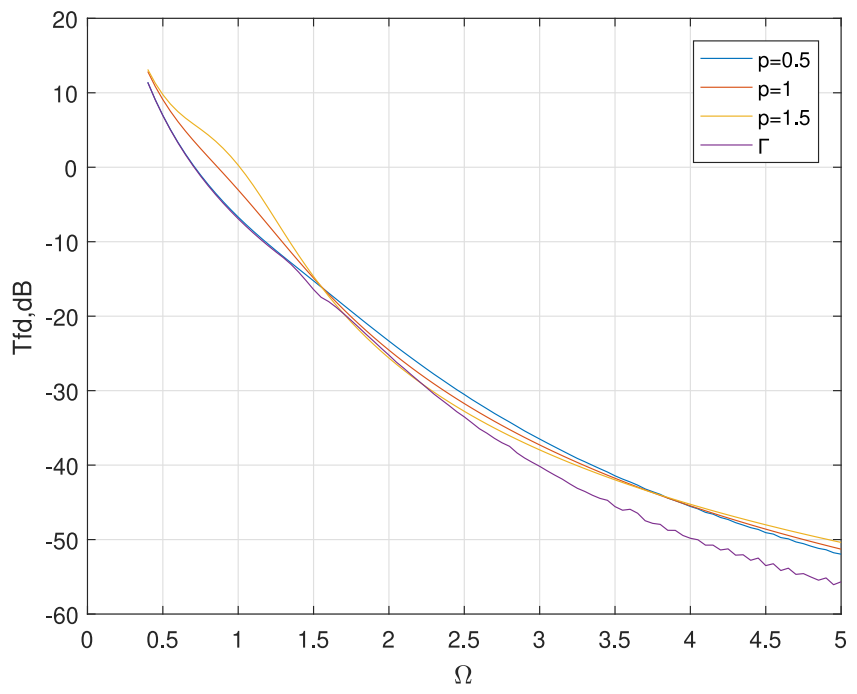

FigURE 12. "Force-displacement" transmission function in the case of Duffing-like equation with fractional damping (2.8) and hysteretic damping (2.18) (a coefficient at the cubic term is $\beta=1)$.

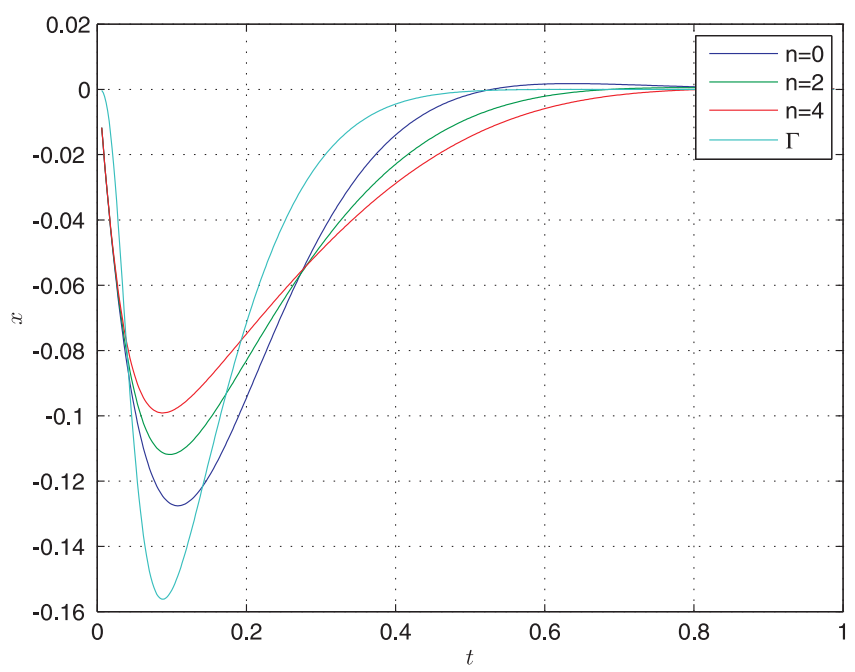

FIgURE 13. The relative motion of a car $x(t)$ under single pulse affection for various kinds of damping.

form:

$$
\ddot{z}(t)+2 \zeta \omega_{0}[1+z(t)]^{n} \dot{z}(t)+\omega_{0}^{2} z(t)=\delta(t) .
$$

For the hysteretic damper we have:

$$
\ddot{z}(t)+\omega_{0}^{2}\left\{\alpha W\left[t, z_{j}(t)\right] z(t)+z(t)\right\}=\delta(t) .
$$

Figure 13 shows the response of the system with viscous and hysteretic dampers to a single pulse. 
As it can be seen from this figure, energy dissipation in the case of hysteretic damper is faster than in the case of linear and nonlinear viscous dampers, as well as in the case of fractional damper. This fact demonstrate (in terms of observable characteristics such as transmission functions) an efficiency of the hysteretic damper.

\section{Conclusions}

This work is considered the various kinds of damping processes that occur in the oscillations of real mechanical systems with damping blocks. Namely, we consider features of the linear and nonlinear viscous damping, as well as fractional damping, and hysteretic damper. In this work we paid special attention to the hysteretic damper, which design model is based on the Ishlinskii-Prandtl model (this model is based on the operator approach to description of the hysteretic nonlinearities; in this way such a model has a great physical meaning in comparison with phenomenological models based on the functional nonlinearities, such as the Bouc-Wen model, etc.). We presented the numerical results for the force transmission function and the "force-displacement" transmission function (observable characteristics of the oscillating system). Phase portraits of the system with various kinds of damping are plotted and analyzed. We have also considered the response of the system with various kinds of damping on the single pulse affection. Obtained results allow to compare the various types of viscous dampers (linear and nonlinear), fractional and hysteretic damper (in terms of transmission functions). Based on the obtained numerical results we can formulate the following concluding note: hysteretic damper based on the Ishlinskii-Prandtl material has a high efficiency both in the resonance region and beyond in comparison with the viscous (linear and nonlinear) damper, as well as the fractional damper. The disadvantage of the hysteretic damper is in decreasing of the ability to reduce the relative movement of the car under external forces outside the resonance region of the system.

Acknowledgements. This work was supported by the RFBR (17-01-00251-a, 18-08-00053-a and 19-08-00158-a). The contributions by M.E. Semenov and P.A. Meleshenko (Hysteretic damping) were supported by the RSF grant No. 19-11-00197.

\section{REFERENCES}

[1] V.I. Babitsky and V.L. Krupenin, Vibration of Strongly Nonlinear Discontinuous Systems. Springer, Berlin, Heidelberg (2001).

[2] J.M. Balthazar, R.M.L.R.F. Brasil, J.L.P Felix, A.M. Tusset, V. Picirillo, I. Iluik, R.T. Rocha, A. Nabarrete and C. Oliveira, Dynamics behaviour of an elastic non-ideal (NIS) portal frame, including fractional nonlinearities. J. Phys: Conf. Ser. 721 (2016) 012004(1)-012004(12).

[3] M. Borowiec, G. Litak and A. Syta, Vibration of the duffing oscillator: Effect of fractional damping. Shock Vib. 14 (2007) $29-36$.

[4] J.L.P Felix, J.M. Balthazar, R.M.L.R.F. Brasil and B.R. Pontes, On lugre friction model to mitigate nonideal vibrations. J. Comput. Nonlinear Dyn. 4 (2009) 034503(1)-034503(5).

[5] P. Hagedorn, On the destabilizing effect of non-linear damping in non-conservative systems with follower forces. Int. J. Non-Linear Mech. 5 (1970) 341-358.

[6] V. Hassani, T. Tjahjowidodo and T. Nho Do, A survey on hysteresis modeling, identification and control. Mech. Syst. Signal Process. 49 (2014) 209-233.

[7] M.A. Krasnosel'skii and A.V. Pokrovskii, Systems with Hysteresis. Springer Verlag, Berlin-Heidelberg-New York-Paris-Tokyo (1989).

[8] Z.Q. Lang, S.A. Billings, R. Yue and J. Li, Output frequency response function of nonlinear Volterra systems. Automatica 43 (2007) 805-816.

[9] M. Latour, Theoretical and Experimental Analysis of Dissipative Beam-to-Column Joints in Moment Resisting Steel Frames. Universal-Publishers (2011).

[10] A.Y.T. Leung, Z. Guo and H.X. Yang, Fractional derivative and time delay damper characteristics in Duffing-van der Pol oscillators. Commun. Nonlinear Sci. Numer. Simul. 18 (2013) 2900-2915.

[11] Q. Lv and Z. Yao, Analysis of the effects of nonlinear viscous damping on vibration isolator. Nonlinear Dyn. 79 (2015) 2325-2332.

[12] A. Luongo, F. D'Annibale and M. Ferretti, Hard loss of stability of Ziegler's column with nonlinear damping. Meccanica 51 (2016) 2647-2663.

[13] A. Luongo and F. D'Annibale, Nonlinear hysteretic damping effects on the post-critical behaviour of the visco-elastic Beck'beam. Math. Mech. Solids (2016). 
[14] A. Luongo and F. D'Annibale, Linear and nonlinear damping effects on the stability of the Ziegler column. Structural Nonlinear Dynamics and Diagnosis: Selected papers from CSNDD 2012 and CSNDD 2014. Edited by M. Belhaq. Springer International Publishing (2015) 335-352.

[15] Q. Ma, G. Cui and T. Jiao, Neural-network-based adaptive tracking control for a class of pure-feedback stochastic nonlinear systems with backlash-like hysteresis. Neurocomputing 144 (2014) 501-508.

[16] Z. Milovanovic, I. Kovacic and M.J. Brennan, On the displacement transmissibility of a base excited viscously damped nonlinear vibration isolator. J. Vib. Acoust. 131 (2009) 054502(1)-054502(7).

[17] A.H. Nayfeh and D.T. Mook, Nonlinear oscillations. John Wiley \& Sons (2008).

[18] Z.K. Peng, G. Meng, Z.Q Lang, W.M. Zhang and F.L. Chu, Study of the effects of cubic nonlinear damping on vibration isolations using harmonic balance method. Int. J. Non-Linear Mech. 47 (2012) 1073-1080.

[19] D. Rachinskii, On geometric conditions for reduction of the Moreau sweeping process to the prandtl-ishlinskii operator. Discr. Continu. Dyn. Syst. B 23 (2018) 3361.

[20] Y. Rochdi, F. Giri, F. Ikhouane, F.Z. Chaoui and J. Rodellar, Parametric identification of nonlinear hysteretic systems. Nonlinear Dyn. 58 (2009) 393-404.

[21] M. Ruderman and D Rachinskii, Use of prandtl-ishlinskii hysteresis operators for coulomb friction modeling with presliding. J. Phys.: Conf. Ser. 811 (2017) 012013.

[22] M.E. Semenov, A.M. Solovyov, P.A. Meleshenko and A.I. Barsukov, Bouc-wen model of hysteretic damping. Proc. Eng. 201 (2017) 549-555.

[23] M.E. Semenov, A.M. Solovyov and P.A. Meleshenko, Elastic inverted pendulum with backlash in suspension: stabilization problem. Nonlinear Dyn. 82 (2015) 677-688.

[24] M.E. Semenov, P.A. Meleshenko, A.M. Solovyov and A.M. Semenov, Hysteretic nonlinearity in inverted pendulum problem. In Structural Nonlinear Dynamics and Diagnosis: Selected papers from CSNDD 2012 and CSNDD 2014, edited by M. Belhaq. Springer International Publishing (2015) 463-506.

[25] J.-Y. Tu, P.-Y. Lin and T.-Y. Cheng, Continuous hysteresis model using duffing-like equation. Nonlinear Dyn. 80 (2015) $1039-1049$.

[26] A.M. Tusset, J.M. Balthazar, D.G. Bassinello, B.R. Pontes and J.L.P. Felix, Statements on chaos control designs, including a fractional order dynamical system, applied to a "MEMS" comb-drive actuator. Nonlinear Dyn. 69 (2012) $1837-1857$.

[27] J. Padovan and J. T. Sawicki, Nonlinear vibrations of fractionally damped systems. Nonlinear Dyn. 16 (1998) $321-336$.

[28] R. Richards, Comparison of linear, nonlinear, hysteretic, and probabilistic mr damper models. Master's thesis, Faculty of the Virginia Polytechnic Institute and State University (2007).

[29] E. Rigaud and J. Perret-Liaudet, Experiments and numerical results on non-linear vibrations of an impacting hertzian contact. part 1: harmonic excitation. J. Sound Vib. 265 (2003) 289-307.

[30] F. Rüdinger, Tuned mass damper with fractional derivative damping. Eng. Struct. 28 (2006) 1774-1779.

[31] A. Syta, G. Litak, S. Lenci and M. Scheffler, Chaotic vibrations of the duffing system with fractional damping. Chaos 24 (2014) 013107(1)-013107(6).

[32] M.E. Semenov, A.M. Solovyov, P.A. Meleshenko and J.M. Balthazar, Nonlinear damping: From viscous to hysteretic dampers. In Recent Trends in Applied Nonlinear Mechanics and Physics, edited by M. Belhaq. Springer International Publishing (2018) $259-275$. 